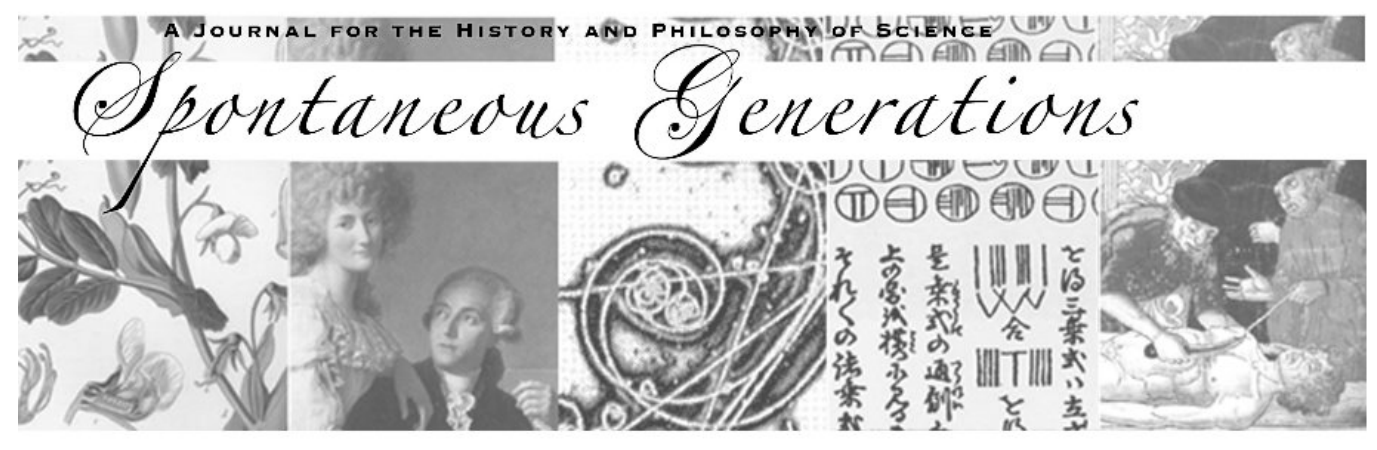

\title{
Equipment for an Experiment
}

Author(s): Rom Harré

Source: Spontaneous Generations: A Journal for the History and Philosophy of Science, Vol. 4, No. 1 (2010) 30-38.

Published by: The University of Toronto

DOI: $10.4245 /$ sponge.v4i1.11334

EDITORIAL OFFICES

Institute for the History and Philosophy of Science and Technology Room 316 Victoria College, 91 Charles Street West

Toronto, Ontario, Canada M5S 1K7

hapsat.society@utoronto.ca

Published online at jps.library.utoronto.ca/index.php/SpontaneousGenerations ISSN 19130465

Founded in 2006, Spontaneous Generations is an online academic journal published by graduate students at the Institute for the History and Philosophy of Science and Technology, University of Toronto. There is no subscription or membership fee. Spontaneous Generations provides immediate open access to its content on the principle that making research freely available to the public supports a greater global exchange of knowledge. 


\title{
Equipment for an Experiment*
}

\author{
Rom Harré ${ }^{\dagger}$
}

\begin{abstract}
Science is as much defined by the local "instrumentarium," the equipment available to an experimenter at a particular time and place, as by its discoveries and theories. Instruments are devices for detecting and measuring natural phenomena, linked causally to those aspects of nature they are used to record. Some are inorganic, made of glass and metal, while others are organic, the bodies and body parts of living or once living plants and animals. In contrast, pieces of apparatus are quite different in the ways in which they work. They too can be organic or inorganic, but their role is to model things, process, structures, and so on in the natural world. They are used to study natural processes by simulating them in a user-friendly way. Keeping the distinction between instruments and apparatus in mind is crucial to understanding the power of the experimental method.
\end{abstract}

We do not know at what moment human beings moved from simply observing the phenomena of nature to actively intervening to increase the store of reliable knowledge-or even if there was such a moment. Equipment is the meeting point between the active experimenter and the potent stuff of the universe. Typically, if one opened the door of a laboratory-for example, like the one in the well-known illustration of the famous room at the Royal Institution where Michael Faraday worked-there would be many things made from glass and metal, wrought into shapes just right for their purposes. At the same time, the bodies of animals and plants, their parts and secretions, were also being pressed into service for sincere purposes. Robert Boyle not only studied the behaviour of gases in glass u-tubes, but also the role of the blood in experimenting on dogs. However, since record-keeping came into practice, there has been plenty of evidence of the use of equipment for all sorts of knowledge-gaining purposes. As philosophers and historians, contemplating the activities of scientists, we ask what those purposes are and how they are achieved.

* Received 11 February 2010.

$\dagger$ Rom Harré taught philosophy of science at Oxford University for many years. Recently, he has turned to the study of philosophical problems in the human sciences. He now divides his time between Georgetown University in Washington DC and the Centre for the Philosophy of Natural and Social Science at the London School of Economics where he works as Director. 
Let us call the equipment available at some time and place to an experimenter the instrumentarium. Faraday's lab, like the school science laboratories of our youth, was filled with inorganic equipment: glass test tubes, a coil of wire, and a magnet, all suitably arranged. An important part of the instrumentarium is and has always been organic, equipment constructed from living materials or materials that were once alive. Spiders, dogs, and chimps were used to test the extraterrestrial environment in the space program. Harry Harlow used live infant monkeys in his experiments on mothering (Harlow 1971). Lichens have been used to measure the age of rocks. Organic indicators, such as litmus paper, are used to detect the presence of acids and alkalis in solutions.

To get a clear picture of the nature and uses of scientific equipment we need to explore the basic distinction between instruments and apparatus. Instruments are related causally to the natural world as detectors of natural phenomena such as litmus solution, which reveals whether a solution is acidic, or thermometers, with which we measure temperature. Some natural phenomenon causes an effect in the instrument, and this effect is the source of an item of scientific knowledge. An apparatus, however, stands in a different relation to the world we are using it to study. From a logical point of view, a piece of equipment, such as a Wheatstone bridge or a flask and gas jar, is a model of some part of the natural world created by abstraction and used to represent some aspect of that world. Causal processes are internal to the apparatus whereas a model is related conceptually to the world. A calorimeter filled with salty water represents the Arctic ocean by mimicking one small segment of it. It is not causally related to that ocean, as the thermometer of an explorer would be.

The distinction between instruments and apparatus is a crucial one for the philosopher or historian looking into the methods of science. These terms are not quite so sharply distinguished in customary usage as I will use them here, yet they roughly delineate a central distinction in the equipment we draw from the instrumentarium. Let us now turn to examine the uses of each of these categories of knowledge-garnering equipment in more detail.

\section{Instruments}

Instruments and natural phenomena are related causally. The state of something being used as an instrument can be affected by the state of something in the world. There are detectors, such as litmus paper sensitive to the presence of acids and alkalis, changing from red to blue in relation to the $\mathrm{pH}$ of the solution; transgenic fish that react to the presence of pollutants in the water, fluorescing in response to the presence of contaminants; and so on. There are also all sorts of measuring devices 
for ascertaining the relative quantities of material things, such as rulers, clocks, balances, and ammeters. For example, thermometers change their state in an orderly manner in response to changes in the temperature of their surroundings by the expansion of the fluids enclosed in the body of the instrument. Some of these instruments are organic or derived from biological sources. For example, the relative sizes of the diameters of discs of lichens have been used to measure the age of glacier moraines.

Changes in the state of the system to be measured must stand in some orderly relationship to observable changes brought about in the measuring device. The displacement of the needle in an ammeter, for example, must be proportional to the amount of current in the circuit in which it is incorporated. The numbers and sequence of different species of fossil midges in the sediment on the floors of lakes can be used to determine past temperatures of those lakes since the temperature at a given time causes one species of gnat to predominate.

Instruments both as detectors and as measurers are thus related causally to the world.

\section{Apparatus}

The world presents itself to an enquirer in a myriad of complex forms. An important part of scientific research is to abstract one process-say a chemical reaction-from complex clusters of processes in nature. Then, in relative isolation and in strictly controlled conditions, this process can be studied without the interference of external causal influences. In a physics laboratory one might find a simple copper vessel containing a mixture of ice, salt, and water: a basic calorimeter. It can be used to reproduce the cooling of salt water below the temperature at which pure water freezes. This happens in the sea in the Arctic winter, though for how long this will go on in nature we do not know thanks to global warming. The conditions of the winter climate at either pole are much more complex than what we can represent with a calorimeter. In the actual sea there are currents and landmasses exerting their particular influences on the formation of the winter ice that floats on the surface of the sea. The calorimeter provides knowledge of one part of the process of the freezing of salt water because of an analogy between the water in the calorimeter and in the ocean. The scientific value of experiments with the calorimeter depends on the balance of differences and similarities between the apparatus and the real-world phenomenon it represents. When designing apparatus, the balance of similarities and differences is crucial.

The chemists of the eighteenth and nineteenth centuries were adept at constructing pieces of apparatus for isolating physical and chemical processes from the complex natural environments in which they occurred. 
Lavoisier set up an apparatus that abstracted the role of atmospheric gases from all the other conditions involved in combustion (Lavoisier 1783). He isolated the process by which a metal was reduced to ash, and then followed the reactions by which the metal was eventually recovered. The value of this research as a source of knowledge of the calcination of metals and the processes involved in smelting ores depended on the fact that Lavoisier had abstracted the core processes of burning and smelting and reproduced them in his laboratory.

The relation between apparatus and world is conceptual. We reason from what happens in the laboratory to how things might be in nature. In building an apparatus to represent a natural process we reason in the reverse direction, again following the pattern of analogy, balancing similarities and differences from reality to analogue. If something happens in a certain way in the laboratory as we run an experiment in a well-designed piece of apparatus, it is likely to happen thus and so in the world which the apparatus represents, all else being equal! Experimenting with apparatus contributes to our scientific knowledge because we believe that it reproduces an instance of a natural regularity that exists in the real world, embedded in a cluster of other processes from which it must be abstracted. In the total pattern of analogy that is expressed in the concrete construction of the apparatus, it is the similarity dimension that supports the extraction of an item of knowledge from the behaviour of the apparatus.

However, the reproduction of natural processes can go further than isolating just one aspect of a complex reality. An important part of advanced laboratory work is the construction of working models of the processes of nature in a conveniently simplified form. In order to study the process of the formation of rainbows, Theodoric of Freiberg set up racks of rows of spherical flasks filled with water (Wallace 1951). Each flask represented a raindrop, and the whole rack was a representation of the curtain of falling rain. Setting a lamp in the same relative position to the rack as the Sun would have been to the water drops in a real rain shower, he was able to produce a working model of a complex natural phenomenon in his laboratory. Here he could do something that would be impossible to do in nature-study the paths taken by rays of light of different colours through the water in flask as a model or analogue of a raindrop. Theodoric drew his conclusions about the way rainbows were generated by reasoning through analogy. The behaviour of the light in the flasks was not caused by a real rainbow-it simulated one.

Theodoric constructed an apparatus of glass and water. It was an inorganic creation. Sometimes an apparatus is largely made up of organic materials, including living things. To study the relation between infant welfare and mothering practices, Harry Harlow built an apparatus 
that simulated this relation using monkeys as analogues of human beings (Harlow 1971). However, the logical structure of this experimental apparatus was more complex: he wanted to isolate specific aspects of mothering and to do so he constructed analogues of monkey mothers. The apparatus consisted of a wire frame with a teat, which represented just one mothering behaviour-providing nutrition. A cloth-covered frame abstracted another aspect of monkey mothering-providing comfort. Was suckling more significant than comfort in the lives of infant monkeys? These abstract analogues enabled him to investigate the question without the interference of all sorts of other aspects of nurturance.

Apparatus is related conceptually to the world it helps us to understand.

SOME WORKING ASSUMPTIONS IN THE USE OF LABORATORY EQUIPMENT

\section{Instruments}

To infer something about an object of interest from the state of pieces of equipment in use for measuring or for detecting invisible and intangible things, substances, and processes-in a laboratory or elsewhere-the scientist must believe that there has been a causal interaction between the world and the instrument, as I have argued. Generally it is assumed that the instrument is in a way detached from or outside of the world it is being used to study. So an important underlying assumption of the use of instruments for detecting or measuring an attribute of an inhabitant of the natural world is that the equipment is affected by but does not affect that which it samples, or least not much. Sometimes this assumption does not hold well and allowances for reciprocal effects must be made. Sometimes the thermometer requires so much heat to expand the mercury that the liquid being studied cools down substantially. Sometimes the experimental procedure kills the animal at the core of the apparatus, as when Pavlov snipped the vagus nerve of one of his dogs to demonstrate nervous control of pancreatic secretions (Pavlov 1897). Skilled experimenters know how to compensate for these exceptions. For example, usually the pressure in the car tires is not significantly reduced by the amount of air needed to activate the tire gauge. Ideally instruments are designed to minimize reciprocal causal reactions. The readings of instruments depend on causal relations that link states of the world to observable states of the instrument for their scientific value, which are essentially one-way, world to instrument.

\section{Apparatus}

Using a piece of laboratory equipment as a model for some natural process is related conceptually rather than causally to the rest of the material world, as I have emphasized. Of course an apparatus is part of 
that world. A map of the world is an object in the world, just as an authentic model village contains a model of itself. Again, there is an isolation assumption involved. The model world contains as much of the real world as it needs to evolve its own forms of natural processes. Although it is set aside from the world, it represents whatever happens in the model as authentic to the target. For example, Clarke's famous study (Clarke 1955) of the effects of overcrowding on reproduction rates followed the changes in the endocrine glands of voles in model populations; from his observations, Clarke drew conclusions about population biology of all sorts of other mammals, including human beings. The model worlds were set up so as to exclude extraneous factors, such as predators and meteorological vagaries. Sometimes the "isolation" assumption is rudely disturbed, as when the vibrations of the early morning trams in Cleveland, Ohio, upset the equipment Michelson and Morley were using to measure the speed of light in different directions. Only rarely does the isolation assumption need to be dealt with explicitly. For the most part, an apparatus as a model can be used as if it were detached from or outside the material world, as if it were a truly isolated system.

Models or analogue representations of aspects of the world under study are extremely common in the sciences. An analogue can be analyzed in terms of likenesses and differences to that which it is analogous to. In the sciences, model-building is usually based on a process that is already well understood and used to illuminate something that is not. The former is the source of the model; the latter, its subject.

Darwin used the breeding of pigeons and farm animals as the source of his model of speciation, the process he imagined had led to the diversity of species, calling it natural selection. The idea of "selection," modeled on breeding practices, the farmer's selection of desirable features in the animals to breed from next season, was an untried analogue of what might be the natural process truly responsible for the variation within and among species. That mysterious process was the subject that Darwin's model was intended to represent (Darwin 1859). This idea of the trio "Source-Model-Subject" proves very useful when we analyze the role of particular pieces of apparatus and assess the quality of their contributions to knowledge.

Some equipment can be thought of as constructed to model a process, structure, or system so that we can understand it via a simplified and manageable material setup. It often happens that the subject of the model cannot easily be manipulated or experimented on, but the model that represents it can be. For example, a culture of a particular bacterium thought to be responsible for an infectious disease can be grown on agar in a Petri dish. An infusion of a possible antibiotic is introduced 
and we watch for an effect on the bacteria of the culture. This is an in vitro version or model of a phenomenon that we have reason to believe occurs in vivo, when an antibiotic is given to a patient with a certain kind of infection. An apparatus such as the simple Petri dish, a model of an infected human being, represents only some aspects of a complex reality, the infective agent and the antibiotic. This kind of modeling-creating a simplified version of a real-world complexity-is a very common scientific technique. We saw its use in calorimetric studies of the density of water around its freezing point. The knowledge-producing power of an apparatus is dependent on a subtle balance between abstraction and verisimilitude. Of course, the balance can only be struck on a case-by-case basis-there are no universal rules for determining the fruitfulness of models.

Studying domesticated versions of natural things, systems, and processes supports quite strong inferences to the way things are in the wild, since the same kind of material systems and phenomena occur in the wild and in domestication. The metaphor of domestic versus feral versions of things and processes applies equally to inorganic and organic processes. The feral targets are, of course, instances of natural kinds, and so, the designer of an apparatus hopes, are the beings activated in the equipment. The art of the experimenter is to select and modify feral beings to create a powerful model of a natural system. Domesticated animals are still animals and can be used to model the genetics of their feral cousins! An apparatus, as a model, however much it is simplified and tidied up as a version of its source, is a piece of nature in the laboratory. Of course, the richness and authority of inferences from model to subject will depend on how relations of similarity and difference between apparatus made by technicians and real-world systems are weighted by the interests of the researcher in performing the experimental manipulations. These are the premises of reasoning by analogy. Since anything can in principle be analogous to anything else, the art of analogical reasoning requires a certain skill and discretion. Modeling is a scientific technique that requires a good deal of intuition and insight to be really effective. Nevertheless, it is the most powerful tool in the instrumentarium available to the experimenter.

Sometimes the modeling analogy leads to hypotheses about what might have happened, or what could be happening, in processes that cannot be observed directly with any experimental apparatus or detector from the current instrumentarium. Creating mixtures of methane and water vapour and passing electric discharges through them in a closed globe has been used to try to simulate possible conditions of the Earth's early atmosphere.

In reflecting on the analogical power of a piece of apparatus we must 
ask whether it is simply behavioural similarities between the apparatus and its subject that matter (what we could call the behavioural analogy) or whether we are ready to go further and infer something about the process, hitherto unobserved, that brings about that behaviour-the material analogy at the heart of a model. The ingenious selection of one of the Galapagos islands as a model world to study natural selection allowed Rosemary and Peter Grant of Princetown University in the nineteen seventies to investigate, by analogy, the processes that were in principle unobservable, that had brought about the changes in the forms of plants and animals over millions of generations. The Grants observed, firsthand, the effects of environmental privation and sexual selection that Darwin had only imagined (Weiner 1995).

\section{THE CONTENTS OF THE INSTRUMENTARIUM}

Looked at in this way, scientific equipment includes four main classes of devices. There are instruments for detecting the presence of something in the environment, such as levels of carbon monoxide. There are measuring instruments, for determining the quantitative values of properties of their targets, such as the movement of a coil in an electric field as an indicator of "electrical pressure." There are pieces of apparatus with which natural processes can be studied in isolation, such as Mendel's garden plots where he worked out the algebraic law of hybridized attributes of peas by studying a few attributes among the many that plants exhibit. Lastly, there are pieces of apparatus that serve as models representing very complex processes and situations in nature in ways that can be more convenient than studying those complexities in the natural world itself. Such apparatus can even created as computer programs, such as the models that are used to predict weather patterns.

Each laboratory has its characteristic instrumentarium, the actual equipment available to an experimenter. Depending on the generosity of the laboratory's budget, the instrumentarium will consist not only of what is in the storeroom, but also what is to be found for purchase in the catalogues of instrument makers. In the great days of experimental science, in the time of Michael Faraday, for example, the laboratory was not only a place where instruments and pieces of apparatus were used, but also where instruments and apparatus were made. Science has always found a place for living beings in the instrumentarium as well as equipment made of inorganic materials. For example, in Pavlov's laboratory the instrumentarium was almost exclusively populated by dogs.

The distinction between instrument and apparatus is fundamental to understanding the experimental method. Equipment that is activated by causal relations with states of nature is fundamentally different in its 
ontological status from that in which causal processes in nature are replicated in simpler forms within the material system of the apparatus.

\author{
ROM HARRÉ \\ Psychology Department \\ Georgetown University \\ Washington, DC 20057 \\ Centre for the Philosophy of Natural and Social Science \\ London School of Economics \\ harre@georgetown.edu
}

\title{
REFERENCES
}

Clarke, John. R. 1955. The influence of numbers on reproduction and survival in two experimental vole populations. Proceedings of the Royal Society 144, no.914: 66-83.

Darwin, Charles. 1859. On the Origin of Species. London.

Faraday, Michael. 1855 [1951]. Experimental Researches in Electricity. London: Everyman.

Harlow, Harry. F. 1971. Learning to Love. San Francisco: Albion.

Harré, Rom. 2009. Pavlov's Dogs and Schrödinger's Cat: Scenes from the Living Laboratory. Oxford: Oxford University Press.

Lavoiser, Antoine L. 1783 [1802]. Essays on the Effects Produced by Various Processes on Atmospheric Air. Trans R. Kerr. London: Creach.

Pavlov, Ivan. 1897. The Working of the Digestive Glands. Birmingham: Classics of Medicine.

Wallace, William A. 1951. The Scientific Method of Theodoric of Freibourg. Freibourg, Germany: Freibourg University Press

Weiner, Judith. 1995. The Beak of the Finch. London: Cape. 